\title{
Analyse des Kontinuums mit surrealen Zahlen
}

\author{
Thomas Morgenstern (D)
}

Eingegangen: 19. Mai 2021 / Angenommen: 2. Januar 2022 / Online publiziert: 26. Januar 2022

(C) Der/die Autor(en) 2022

Zusammenfassung Ausgehend vom anschaulichen Begriff des Kontinuums betrachten wir drei klassische Konzepte des Kontinuums und entwickeln für das lineare, geometrische Kontinuum mathematische Modelle, wobei wir vereinfacht eine Strecke betrachten. In allen drei Fällen sind die Teile des Kontinuums linear angeordnet und ihre Ordnung ist dicht.

Das Kontinuum des Parmenides ist eine unteilbare Einheit. Das entsprechende Modell der Strecke ist eine Einermenge und somit ohne Lücken.

Das Kontinuum des Aristoteles ist potentiell unendlich, aktual endlich oft teilbar und die Teile sind wieder teilbare Kontinua. Das entspreche Modell der Strecke ist isomorph zum angeordneten Körper der rationalen Zahlen.

Das hypothetische Kontinuum des Zenon ist aktual unendlich oft teilbar und die Teile sind wieder teilbare Kontinua. Die Teile der Strecke werden mittels transfiniter Rekursion durch fortgesetzte Zweiteilung konstruiert und tragen damit in natürlicher Weise die Struktur eines geordneten, vollen, dyadischen Wurzelbaums.

Gleichzeitig wird ein Isomorphismus der Punkte zum nicht-archimedischen angeordneten Körper der surrealen Zahlen konstruiert, dem universellen, absoluten, arithmetischen Kontinuum. Dann gibt es viele infinitesimal kleine und infinit große Strecken. Die Punkte bilden eine echte geordnete Klasse mit intervallvollständiger, aber nicht schnittvollständiger Ordnung, die sehr viele Lücken hat.

Schlüsselwörter Kontinuum · Parmenides · Aristoteles · Zenons Paradoxie der Vielheit · Surrealen Zahlen · Infinitesimale

Mathematics Subject Classification (2010) $00 \mathrm{~A} 30 \cdot 03 \mathrm{~A} 05 \cdot 03 \mathrm{E} 10$

Thomas Morgenstern $(\varangle)$

Hochschule Karlsruhe, Moltkestraße 30, 76133 Karlsruhe, Deutschland

E-Mail: Thomas.Morgenstern@h-ka.de 


\section{Einleitung}

„Was ist das Kontinuum?“ diese Frage wird seit den Anfängen der antiken griechischen Philosophie und Mathematik kontrovers diskutiert [3, 5, 19]. Kontinua sind homogen und nicht diskret, darin stimmen alle überein [19, S. 6].

Für Parmenides gilt: „das Seiende ist eins“, ein unteilbares, teilloses Ganzes [20, S. 112ff.]. Daraus würde folgen, dass Veränderung und Bewegung bloßer Schein sind. Dies widerspricht dem offenkundigen Anschein [20, S. 62f.].

Die Paradoxien Zenons widerlegen die allgemein akzeptierten Meinung „Es gibt viele Dinge“ [17, S. 90]. Nach Platons Darstellung sei: „[Zenons Schrift] eine Art Unterstützung für die These des Parmenides [es gibt nur Eines] gegen diejenigen, die versuchen, sie lächerlich zu machen“. Durch eine reductio ad absurdum zeigt Zenon, ,aus deren Annahme ,wenn (die Dinge) viele sind“" ergeben sich, wenn man sie hinreichend durchgeht, wohl noch viel absurdere Folgerungen als aus der Annahme, dass (alles) eins sei.“ [20, S. 49], [17, S. 89f., S. 291ff.], [19, S. 10f.].

In seiner Physikvorlesung stellt Aristoteles die Frage nach dem Wesen von Ort, Zeit und Bewegung, deren gemeinsame und allgemeine Bestimmung Kontinuität ist [1, Buch VI], [21, S. 254]. Aristoteles behandelt das Kontinuum als Grundstruktur der vorgegebenen anschaulichen Welt und untersucht dabei die und nur die Bestimmungen des Kontinuums, die für die Physik notwendig sind. Die Struktur der Kontinuität wird für Aristoteles dann relevant, wenn man Bewegung und Zeit gegenseitig misst, erst dabei zeigt sich ihre unbegrenzte Teilbarkeit. [21, S. 254ff.], [19, S. 15f.].

$\mathrm{Zu}$ Beginn des letzten Jahrhunderts schien unsere Frage für das mathematische Kontinuum durch die bahnbrechenden Arbeiten Hilberts zu den Grundlagen der Geometrie abschließend geklärt zu sein: das arithmetische Kontinuum waren die reellen Zahlen $\mathbb{R}$ und das geometrische lineare Kontinuum ihr isomorphes Bild [14]. Auch diese allgemein akzeptierte Meinung wird heute hinterfragt - die Frage ist wieder offen $[2-4,10,11,15]$.

Bei Hilberts formal-axiomatischer Methode werden zudem die geometrischen Grundbegriffe, wie „Punkt“ oder „Gerade“, gar nicht definiert, sie sind nur Variablen; die Theorie bleibt inhaltlich leer ohne Gegenstände - lediglich reine, formale Gedanken. Die formale Methode hat Vorteile, denn die Wahrheit der Axiome muss nicht behauptet werden, die Theorie gestattet unterschiedliche Interpretationen und die Widerspruchsfreiheit des Axiomensystems kann, beispielsweise durch Angabe eines Modells, untersucht werden.

Der geübte Mathematiker hat schon eigene Intuitionen entwickelt und kann auf dieser Grundlage rein abstrakt-formal arbeiten; der Lernende muss die Grundbegriffe allerdings noch durch eigene Anschauungen füllen. Auch hierbei dient Hilbert als Leitbild. Als Schüler hatte Hilbert in der Oberprima einen Geometrieunterricht auf hohem Niveau und sein sorgfältig geführtes ,Schulheft zur Neuen Geometrie“ [14, Anhang 327ff.] zeichnet die Linien vor, denen er auch als Lehrer in seinen Vorlesungen folgte: anschauliche Geometrie vor axiomatischer und dann analytischer Geometrie [14, Anhänge S. 275f., 285f.].

Wir gehen daher in dieser Arbeit möglichst anschaulich vor, mathematische Begriffe werden konstruiert, bildlich veranschaulicht und erst anschließend formal de- 
finiert. Der formale Schwerpunkt liegt hierbei auf den für die Mathematik und Informatik so wichtigen rekursiven Definitionen und induktiven Beweisen. Der an der Lehre interessierte Leser wird dabei alle unendlich großen und infinitesimal kleinen Zahlen durch eine einfache, einheitliche Rekursionsvorschrift konstruiert und auf einem Zahlenstrahl abgebildet finden, wobei die Wahl des Maßstabs durch Abbildungen motiviert wird [7].

In diesem Zusammenhang sei auch besonders auf den mathematischen Roman von Knuth hingewiesen - nicht nur eine Einführung in die surrealen Zahlen, sondern auch eine Übung in kreativem Denken und forschendem Lernen für mathematisch Begabte [16, Nachwort].

Philosophisch legen wir den klassischen Begriff des anschaulichen, linearen geometrischen Kontinuums zugrunde und versuchen diesen Begriff rekonstruktiv besser zu begreifen. Die Methode der logischen Rekonstruktion ist mehrfach auf die betrachteten klassischen Textstellen angewandt worden; beispielsweise auf das Lehrgedicht des Parmenides [20, S. 177f., 203] oder auf Zenons Argumente gegen die Vielheit [17, Abschn. 5.5]. Die genauen Argumentationsketten und auch die implizit gemachten Voraussetzungen bleiben jedoch unsicher und die Beweise Zenons gelten vielen als inkorrekt. Wir wollen daher versuchen mathematische Modelle zu konstruieren, die zu den Aussagen der Textstellen passen und sie verdeutlichen können.

Uns interessiert in erster Linie: Welchen mathematischen Rahmen müssen wir wählen und welche Voraussetzungen müssen wir machen, wenn wir das Kontinuum durch begrenzte oder unbegrenzte Zweiteilung mathematisch analysieren wollen? Wie weit gelingt das? Wie viele Punkte gibt es? Welche (formalen) Schwierigkeiten tauchen auf? Gibt es auch paradoxe Folgerungen?

Platons Parmenides beschreibt das zutreffend: „Womit also sollen wir beginnen und welche Annahme sollen wir zuerst treffen? Oder wollt ihr, da dieses anstrengende Spiel nun ja gespielt werden soll, dass ich bei mir selbst anfange und mit meiner Annahme, indem ich hypothetisch über das Eine selbst spreche (und aufzeige), was sich logisch ergeben muss, wenn es eins ist und wenn es nicht eins ist?“ „Auf jeden Fall“", habe Zenon gesagt [17, S. 98, 137a7-b5].

\section{Ordnungstheoretische Grundlagen}

Die Teile eines geometrischen Kontinuums sind räumlich trennbar und angeordnet, auch darin sind sich unsere klassischen Autoren einig. Zenon stellt fest: „Wenn es aber ist, muss jedes einzelne eine gewisse Ausdehnung und Dicke haben und muss das eine von ihm an anderer Stelle liegen als das andere. Dieselbe Überlegung gilt für das weiter vorn Liegende." [17, DK B1 S. 257]. Aristoteles beginnt daher seine Betrachtungen des Kontinuums in der Physikvorlesung mit der Bestimmung der Grundbegriffe einer Ordnung [1, 226 b 18ff.]: die Teile des Kontinuums haben unterschiedliche Orte [1, $226 \mathrm{~b}$ 22], [1, $231 \mathrm{~b}$ 4f.] und folgen aufeinander [1, $226 \mathrm{~b}$ 26f.], [1, 231 a 21f.], [21, S. 261].

Bemerkenswert ist, dass bei Euklid Anordnungsaxiome fehlen; Hilbert dagegen fügt entsprechende Axiome früh zu seinem Axiomensystem hinzu [14, Kap. I 33 , 
Axiomgruppe II]. Auch wir wollen das mathematische Kontinuum mit ordnungstheoretischen Begriffen beschreiben - metrische Strukturen ziehen wir möglichst spät heran, um die Messbarkeit nicht für den Begriff selbst schon vorauszusetzen, am deutlichsten wird das beim Begriff der ,Zeit“.

\subsection{Grundlegende Begriffe}

Als Rahmen wählen wir die moderne Logik und Mengenlehre - genauer die allgemeine Mengenlehre und Klassenlogik [18], [7, Anhang zum Teil Null]. Alle Mengen sind Klassen, es gibt aber auch echte Klassen, die keine Mengen sind, beispielsweise die Klasse On der Ordinalzahlen.

Eine strikte partielle Ordnung < auf einer Klasse $A$ ist eine transitive und asymmetrische Relation auf $A$. Eine strikte totale Ordnung oder strikte lineare Ordnung $<$ ist eine konnexe strikte partielle Ordnung (d.h. es gilt $x<y$ oder $y<x$ oder $x=y$ für alle $x, y \in A$ ). Eine strikt geordnete Klasse $(A,<)$ ist eine Struktur bestehend aus einer Klasse $A$ zusammen mit einer strikten linearen Ordnung $<[18$, $\S 8]$.

Ein Dedekind-Schnitt in einer strikt geordneten Klasse $(A,<)$ ist ein Paar $(L \mid R)$ von Teilklassen $L, R \subseteq A$, die eine disjunkte Zerlegung von $A$ definieren (d.h. $L, R \neq \varnothing, L \cup R=A$ und $L \cap R=\varnothing$ ), für alle $x \in L$ und $y \in R$ gilt $x<y$ und $\sup (L) \in L$ oder $\inf (R) \in R$, falls existent.

Unmittelbar benachbarte Teile eines Kontinuierlichen sollen sich berühren, d.h. zwischen ihnen soll es keine Unterbrechungen oder andere Teile derselben Art geben [21, S. 257]; das beschreiben wir mathematisch als Sprungstelle einer Ordnung. Ein Schnitt $(L \mid R)$ heißt Sprungstelle der Ordnung, wenn es Elemente $a \in L$ und $b \in R$ gibt, mit $a=\sup (L), b=\inf (R)$ und $a<b$. Eine lineare Ordnung $(A,<)$ heißt dicht genau dann, wenn es zu jedem $a \in A$ und $b \in A$ mit $a<b$ ein $x \in A$ mit $a<x<b$ gibt. Demnach sind dicht und keine Sprungstellen äquivalent [8, S. 96].

Jeder Teil des Kontinuums hat äußere Grenzen. Die Teile des Kontinuums sollen zusammenhängen, d.h. die entsprechenden äußeren Grenzen benachbarter Teile sollen zusammenfallen und eine Einheit bilden [21, S.259]; ist dies nicht der Fall, dann gibt es dort eine Lücke. Ein Schnitt $(L \mid R)$ heißt Lücke einer strikt geordneten Klasse $(A,<)$ falls $\sup (L)$ oder $\inf (R)$ nicht existieren. $(A,<)$ heißt vollständig, wenn jede nicht leere nach oben beschränkte Teilklasse $L \subseteq A$ ein Supremum und jede nicht leere nach unten beschränkte Teilklasse $R \subseteq A$ ein Infimum besitzt. Demnach sind vollständig und keine Lücken äquivalent [8, S. 98]. Wir sprechen auch von Schnittvollständigkeit und dem Dedekindschen Stetigkeitsaxiom.

Wir werden noch ein zweites ordnungstheoretisches Konzept der Stetigkeit betrachten, dass sich auf Cantor bzw. Veronese zurückführen lässt [6, S. 224], [11, S. 508f.]. In einer metrischen Geometrie sind beide Konzepte gleichwertig.

Eine Intervallschachtelung ist eine Folge $\left(I_{n}=\left[a_{n}, b_{n}\right]\right)_{n=0,1, \ldots}$ abgeschlossener Intervalle bei der $I_{n} \subseteq I_{m}$ für alle $m \leq n$ gilt. Eine Intervallschachtelung konvergiert genau dann, wenn es keine $a<b$ mit $[a, b] \subseteq I_{n}$ für alle $n=0,1, \ldots$ gibt. Eine strikte geordnete Klasse $(A,<)$ heißt intervallvollständig genau dann, wenn es für jede konvergente Intervallschachtelung ein $c \in A$ mit $c \in I_{n}$ für alle $n=0,1, \ldots$ gibt. 
Wir sprechen von Intervallvollständigkeit und der ordnungstheoretischen Version des Cantorschen Stetigkeitsaxioms. Kurzgefasst: $(A,<)$ ist intervallvollständig genau dann, wenn für jede Intervallschachtelung $\bigcap_{n=0,1, \ldots} I_{n} \neq \varnothing$ gilt.

Definition 1 Eine nicht leere, vollständige, dichte, linear geordnete Klasse $(K,<)$ heißt ordnungstheoretisches Modell des linearen Kontinuums.

\subsection{Grundlegende Ergebnisse}

Damit haben wir die ordnungstheoretischen Modelle schon stark eingeschränkt, denn es gilt [8, S. 96, 99]:

Satz 1 Ist $(X,<)$ eine abzählbar unendliche, unbeschränkte, dichte, linear geordnete Menge, dann ist sie ordnungs-isomorph zu $(\mathbb{Q},<)$.

Eine Teilmenge $Q \subseteq X$ heißt dicht in $(X,<)$ genau dann, wenn es zu allen $a, b \in X$ ein $q \in Q$ mit $a<q<b$ gibt. Gibt es in $X$ eine abzählbare, dichte Teilmenge, dann heißt $(X,<)$ separabel.

Satz 2 Ist $(X,<)$ eine unendliche, unbeschränkte, vollständige, dichte, separable, linear geordnete Menge, dann ist sie ordnungs-isomorph zu $(\mathbb{R},<)$.

\subsection{Baumstrukturen}

Eine wohlgeordnete Klasse $(A,<)$ ist eine Struktur, bestehend aus einer Klasse $A$ zusammen mit einer strikten linearen Ordnung $<$, die fundiert ist (d.h. jede nichtleere Teilmenge $\varnothing \neq X \subseteq A$ besitzt ein minimales Element $m=\min (X))$ und die Ordnung genügt der Abschnittsbedingung (d.h. $\{y \in A \mid y<x\}$ ist für alle $x \in A$ eine Menge) [18, §24].

Ein (gerichteter) Wurzelbaum ist eine Struktur $T=\left(A,<_{S}\right)$, bestehend aus einer Klasse $A$ (den Ecken) und einer strikten partiellen Ordnung $<_{S}$ auf $A$, für die es

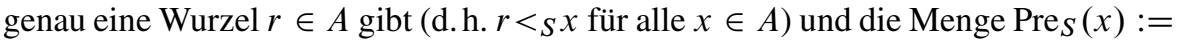
$\left\{y \in A \mid y<_{S} x\right\}$ der Vorgänger von $x$ ist für alle $x \in A$ wohlgeordnet (insbes. linear geordnet); $<_{S}$ heißt auch Baumordnung von $T$ [9, S. 15], [8, S. 303ff.].

$B \subseteq A$ heißt Kette in $A$ genau dann, wenn die auf $B$ eingeschränkte Ordnung eine strikte totale Ordnung ist. $c \in A$ heißt unmittelbarer Nachfolger der Kette $B$, wenn $b{ }_{S} c$ für alle $b \in B$ gilt und es kein $a \in A$ mit $b{ }_{S} a{ }_{S} c$ für alle $b \in B$ gibt. (Für $B=\{b\}$ nennen wir $c$ auch den direkten Nachfolger der Ecke $b$.) $c \in A$ heißt Limeselement der Kette $B$, wenn $c$ unmittelbarer Nachfolger der Kette $B$ ist und es kein $b \in B$ gibt, so dass $c$ unmittelbarer Nachfolger von $b$ ist.

Ein Wurzelbaum $T=\left(A,<_{S}\right)$ heißt dyadischer (binärer) Wurzelbaum genau dann, wenn alle Ecken $a \in A$ höchstens zwei unmittelbare Nachfolger haben und jede Kette $B$ ohne maximales Element höchstens ein eindeutig bestimmtes Limeselemente $c$ als Nachfolger hat. Der dyadische Wurzelbaum heißt voll, falls maximal viele direkte Nachfolger existieren (die geordnete Klasse der unendlichen binären Folgen $\left(\mathrm{Seq}_{2},<_{S}\right)$ aus Definition 6 ist der kanonische volle dyadische Wurzelbaum) [10, S. 243f.], [11, S. 526f.]. 
Ein lexikographisch geordneter Wurzelbaum $T=\left(A,<_{S},<\right)$ ist ein Wurzelbaum zusammen mit einer strikten totalen Ordnung $<$ auf $A$, die die symmetrische Reihenfolge zur Baumordnung $<_{S}$ ist; $d$.h. für alle $c \in A$ definieren wir zwei Mengen $L_{c}:=\left\{x \in \operatorname{Pre}_{S}(c) \mid x<c\right\}$ und $R_{c}:=\left\{x \in \operatorname{Pre}_{S}(c) \mid c<x\right\}$ und in der symmetrischen Reihenfolge gilt $a<b$ genau dann, wenn genau einer der folgenden drei Fälle gilt: $a \in L_{b}$ oder $b \in R_{a}$ oder $R_{a} \cap L_{b} \neq \varnothing$ [11, S. 527].

\section{Das unteilbare Kontinuum}

Das eine Seiende des Parmenides ist das erste Kontinuum, zu dem wir ein passendes mathematisches Modell suchen. „Seiendes nämlich ist dem Seienden nahe.“ [20, B8.25]. „Doch da eine äußerste Grenze es gibt, ist es vollendet von allen Seiten her, ähnlich der Masse einer wohlgerundeten Kugel, inmitten überall gleich; denn nicht etwas größer, noch etwas kleiner ist Not, dass es ist, da oder dort." [20, B8.42-45]. Das eine Seiende hat also keine Löcher, Sprünge oder Lücken, es ist vollständig, homogen und man kann es sich vorstellen wie eine perfekte Kugel.

„Auch ist es nicht teilbar, da gleich in jeder Beziehung.“ [20, B8.22], [19, S. 8f.]. Für Parmenides gibt es also nur ein einziges teilloses und unteilbares Ganzes; kurz gesagt: ,es gibt nur Eines“ [20, S. 112ff.], [17, S. 97, 100, 292], [19, S. 7ff.]. Das dem gemäß Definition 1 entsprechende ordnungstheoretische Modell ist die Einermenge zusammen mit der leeren Relation:

$$
(K,<)=(\{\bullet\}, \varnothing)=(\{\varnothing\}, \varnothing)=(\{(\quad)\}, \varnothing)
$$

$\{\varnothing\}$ ist die 1 der natürlichen Zahlen gemäß von-Neumann $[18, \S 19]$ und $\{(\quad)\}$ die Einermenge der leeren Folge, die wir weiter unten verwenden werden.

\section{Das teilbare Kontinuum}

Das abstrakte Kontinuum ist strukturlos und ohne Möglichkeit Teile zu unterscheiden. Aber das Kontinuum kann zur Gewinnung von zusätzlichen quantitativen und metrischen Informationen begrifflich gegliedert, eingeteilt und strukturiert werden. Die Aufgabe, der sich Aristoteles stellt, ist die Analyse des Kontinuums durch Teilung unter Vermeidung der Zenonschen Paradoxien [15, S. 153], [19, S. 18].

\subsection{Die Teile und das Ganze}

Kontinuität ist für Aristoteles ein Relationsbegriff, der die Eigenschaft des Ganzen, ein Kontinuum zu sein, bestimmt: Kontinuierlich ist etwas, dessen Teile in der Relation der Kontinuität zueinander stehen [21, S. 259f.]. Das Interesse gilt dabei nicht den Teilen als solchen, sondern der Teilung - nur von der Teilung her versteht Aristoteles das Kontinuum, das als solches schon primär vorgegeben sein muss [21, S. 263], [19, S. 19f.]. 
Die Teile hängen zusammen, sind von der gleichen Art wie das Ganze und können nicht unteilbar sein [1, 231 a 24]. Die Teile eines Kontinuums sind somit selbst Kontinua und wieder beliebig teilbar [21, S. 260, 263f.], [15, S. 4], [5, S. 190, 197f.], [19, S. 15ff.]. So kommt Aristoteles zur Bestimmung des Kontinuums: Teilbar in immer wieder Teilbares [1, 231 b 16], [21, S. 262], [19, S. 17]. Wir formulieren:

Definition 2 (Teilbarkeitsprinzip) Die Teile der Teilung eines Kontinuums sind immer wieder teilbare Kontinua.

\subsection{Die Grenzen}

Parmenides schreibt: ,als Gegensatz hoben sie einen Körper heraus und setzten Zeichen voneinander getrennt“" [20, B8.55]. Zeichen (semeion) sind Kennzeichen und Markierungen, die zu einen bestimmten Zweck gesetzt werden [20, S. 179]. Durch Zeichen, die gesetzt werden, lassen sich Teile des Kontinuums unterscheiden. Auch für Aristoteles ist das Kontinuum durch Grenzen teilbar und einteilbar [15, S. 158], [19, S. 23f.].

Es gibt Grenzen, die das Kontinuum außen begrenzen, und innere Grenzen, die zur Einteilungen beliebig gesetzt werden können, die Teile begrenzen, markieren und trennen [3, S. 71], [19, S. 23]. Die Grenzen sind von anderer Art und keine Teile des Kontinuums. Zwischen benachbarten Teilen des Kontinuums gibt es keine weiteren Teile der gleichen Art, sie berühren sich und ihre entsprechenden Grenzen fallen zusammen und bilden eine Einheit [1, 231 a 22 f.], [21, S. 257], [19, S. 15].

\subsection{Linien und Punkte}

Wir sind hier an mathematischen Theorien des linearen, geometrischen Kontinuums interessiert (beispielsweise Linien, Kurven, Strecken, Geraden).

Die Zeichen die einen geometrischen Ort bezeichnen heißen Punkte [2, S. 1, 13f.]. Die Grenzen geometrischer Kontinua sind also Punkte. Eine Linie wird durch zwei äußere Punkte begrenzt. Auch Euklid schreibt: „Ein Punkt (semeion) ist, was keine Teile hat.“ (insbesondere sind Punkte keine Kontinua) und „Die Enden einer Linie sind Punkte.“ [5, Kap. 3 Abschn. 3, S. 200f.]. Innere Grenzpunkte von Linien werden konstruiert, hier berühren sich die Teile einer Linie und sind in einem gemeinsamen Punkt verbunden [1, 227 a 10f.]. Punkte teilen Linien, zerteilen sie allerdings nicht notwendig. Linien können für Aristoteles nicht aus Punkten bestehen [1, 231 a 25f.], [2, S. 4], [3, S. 71f.], [5, Kapitel 3, Abschn. 3.3, S. 204f.].

Auf den Punkt gebracht: Punkte sind gesetzte Zeichen für geometrische Orte, an denen Linien beginnen, enden oder geteilt werden können und ihre Teile sich berühren; aber die Teile von Linien sind nicht Punkte sondern Linien. Dieser Auffassung des Aristoteles wollen wir hier folgen. 


\section{Endliche Teilungen}

Das einfachste lineare, geometrische Kontinuum ist eine Strecke. Eine Strecke wird durch zwei Endpunkte begrenzt und zwischen den zwei Endpunkten der Strecke gibt es stets mindestens einen weiteren inneren Punkt. Bei der endlichen Teilung von Strecken an inneren Punkten entstehen wieder Strecken. Bei Hilbert entspricht das [14, Axiom II.2], [14, §3 Erklärung] und [14, §4 Satz 3]. Die Teile müssen nicht notwendig gleich groß sein, denn die Teilungspunkte können beliebig gesetzt werden. Die Teile und die ganze Strecke ähneln sich jedoch immer [19, S. 17f., S. 23].

\subsection{Zweiteilung}

Wir teilen eine Strecke an einem beliebigen inneren Punkt in zwei Teilstrecken. Durch fortgesetzte endliche Teilung können wir eine Gesamtstrecke dann rekursiv in beliebig viele Teilstrecken teilen. Um diesen Teilungsprozess zu beschreiben, müssen wir uns auf die Teilstrecken und Punkte beziehen und diese dafür benennen - auch dieser Gedanke findet sich schon bei Parmenides: „deshalb wird alles Name sein“ [20, B8.38]. Wir werden Zahlen oder Zeichenketten als Namen verwenden, wobei wir die Gesamtstrecke mit ihren äußeren Grenzpunkten $A$ und $Z$ durch $[A Z]$ bezeichnen (Abb. 1).

Teilstrecken $t_{a}$ bezeichnen wir durch Folgen $a=\left(a_{i}\right)_{i \in[0, n)}$ von Symbolen $a_{i} \in$ $\{0,1\}$ (d.h. Abbildungen $a:[0, n) \rightarrow\{0,1\}$ ). Die Länge dieser Folge ist $l(a)=n$. Die ungeteilte Strecke $[A Z]$ bezeichnen wir auch durch die leere Folge $a=(\quad)$ der Länge $l(a)=0$, d.h. $\left.t_{(}\right):=[A Z]$.

Wählen wir einen Punkt $O$ zwischen $A$ und $Z$ [14, Kap. I $\$ 4$ Satz 3], dann lässt sich die Strecke in zwei Teile teilen; in einen weiter vorn (links) liegenden Teil $[A O]$, den wir mit $(0)$ bezeichnen $t_{(0)}:=[A O]$ und einen weiter hinten (rechts) liegenden Teil $[O Z]$, den wir mit (1) bezeichnen $t_{(1)}:=[O Z]$. Wir ordnen die Teile durch die Relation $t_{(} \quad<_{S} t_{(0)}$ und $t_{(} \quad{ }<_{S} t_{(1)}$ (die Teile $t_{(0)}$ und $t_{(1)}$ sind in der Ordnung $<_{S}$ nicht vergleichbar). Eine zweite, totale Ordnung erhalten wir mit $t_{(0)}<t_{(} \quad{ }<t_{(1)}$.

Wählen wir eine teilbare, bisher nicht geteilte Strecke $t_{a}=t_{\left(a_{i}\right)_{i \in[0, n)}}$ aus, dann gibt es zwei Endpunkte $Q$ und $S$ dieser Strecke und einen Schnittpunkt $P$ zwischen diesen Punkten (Abb. 2). Der Anfangspunkt der Strecke $t_{a}$ sei der Punkt $Q$ (entweder der Punkt $A$ oder der Endpunkt $Q$ einer Strecke $t_{b}$ mit $t_{b}<t_{a}$ ); wir bezeichnen die Strecke $[Q P]$ mit der Folge $(a 0)$, die entsteht, indem wir $a$ durch 0 verlängern, d. h. $(a 0)(i)=a(i)$ für $i \in[0, n)$ und $(a 0)(n)=0$. Der Endpunkt der Strecke $t_{a}$ sei $S$

Abb. 1 Die ganze Strecke [AZ]

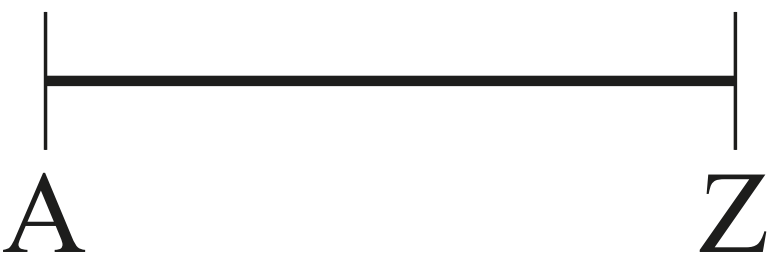




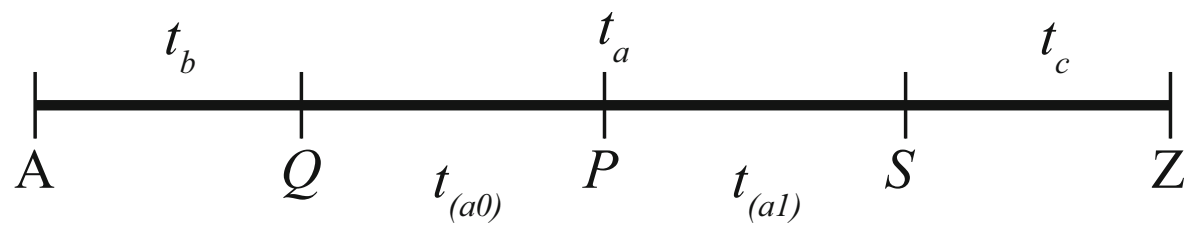

Abb. 2 Teilung der Strecke $t_{a}$

(entweder der Punkt $Z$ oder der Anfangspunkt $S$ einer Strecke $t_{c}$ mit $t_{a}<t_{c}$ ); wir bezeichnen die Strecke $[P S]$ mit der Folge $(a 1)$. Die neuen Teilstrecken ordnen wir in die Baumordnung durch $t_{a}<_{S} t_{(a 0)}$ und $t_{a}<_{S} t_{(a 1)}$ ein und in die totale Ordnung durch

$$
t_{b}<t_{(a 0)}<t_{a}<t_{(a 1)}<t_{c}
$$

für alle Teilstrecken mit $t_{b}<t_{a}$ und $t_{a}<t_{c}$.

Rekursiv definieren wir so einen dyadischen Wurzelbaum mit den Teilen der Teilung als Ecken, der Gesamtstrecke als Wurzel $t_{(}$) und der Teil-Relation $<_{S}$ als Baumordnung. Zusammen mit der totalen Ordnung $<$ ist dies ein geordneter Wurzelbaum (siehe Abschn. 2.3 und Abb. 3).

Die Höhe eines Knotens $a$ über der Wurzel des Baumes ist die Länge $h(a)=l(a)$ der Folge, mit der die Teilstrecke bezeichnet wurde (wie in der Informatik üblich, stellen wir die Wurzel eines Baumes oben und die Blätter unten dar). Die Ordnung der Knoten auf gleicher Höhe $h(a)=n \in \mathbb{N}$ ist die lexikographische Ordnung der Binärfolgen.

\subsection{Das Modell der endlichen Teilungen}

Ist die Menge der Teilstrecken einer endlich großen Strecke endlich, beispielsweise wenn nur eine maximale Anzahl $n$ von Teilungsschritten möglich ist, dann erhalten wir ein diskretes Modell des Kontinuums mit Sprungstellen der Ordnung und mit endlich großen unteilbaren Strecken, was dem Teilbarkeitsprinzip (Definition 2) widerspricht.

Ist die Anzahl der Teilungsschritte endlich aber beliebig, dann gibt es unendlich viele geometrisch unterschiedliche Teilstrecken, wie Zenon folgert: ,Wenn (die Dinge) viele sind, sind sie unendlich viele.“ [17, DK B3 S. 257]. Aristoteles vermeidet Zenons Paradoxie der Vielheit, indem er den Begriff des Unendlichen genauer bestimmt. Zweiteilungen sind theoretisch unbegrenzt ad infinitum durchführbar, zumindest praktisch jedoch nicht. Von den potentiell unendlich vielen Teilen können nur endlich viele aktual verwirklicht sein [5, Abschn. 3.2.2], [19, S. 21].

Die Methode der Zweiteilung liefert abzählbar unendlich viele Teilstrecken, die wir wieder durch Codes $a=\left(a_{i}\right)$ mit $a_{i} \in\{0,1\}$ für alle $i \in[0, \ldots, n)$ bezeichnen. Die rekursiv definierte Ordnung der Teilstrecken ist dicht, denn sind $t_{b}<t_{a}$ Teilstrecken, dann sind beide teilbar und für die durch Teilung von $t_{a}$ erhaltene Teilstrecke $t_{(a 0)}$ gilt $t_{b}<t_{(a 0)}<t_{a}$ (siehe Abb. 2). „Denn es gibt immer wieder 


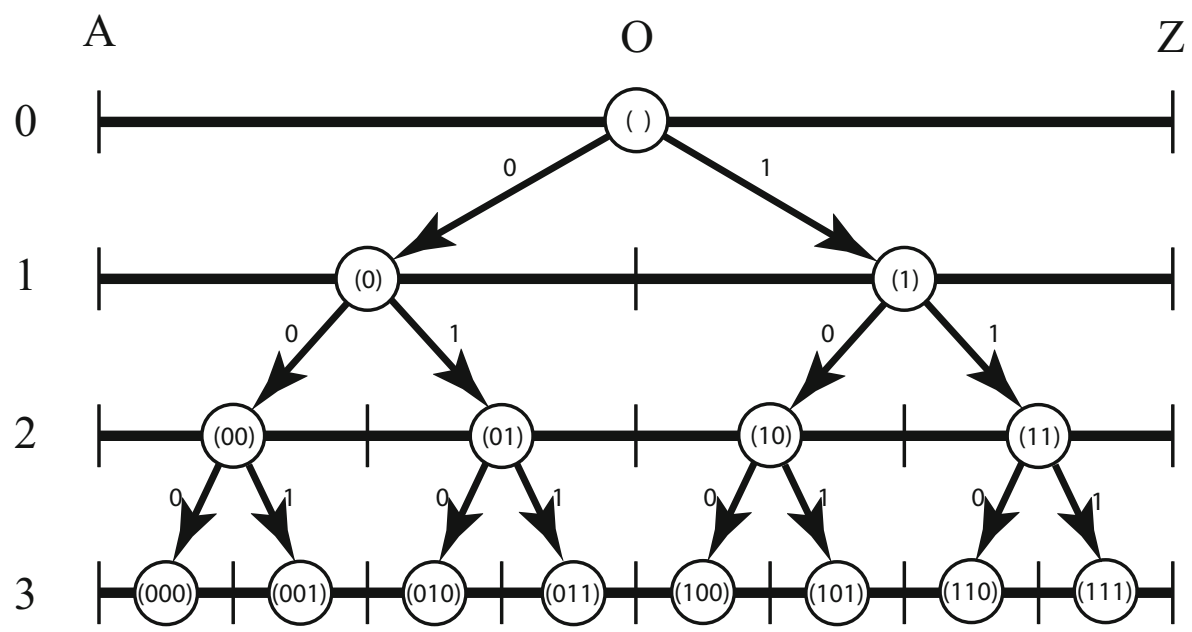

Abb. 3 Baum der Teilstrecken

andere zwischen den Dingen und wiederum andere zwischen jenen." sagt Zenon zur Begründung [17, DK B3 S. 257].

Es gibt bezüglich dieser Ordnung kein minimales Element, keine erste Teilstrecke, auch das stellt Zenon schon fest: „etwas von ihm wird weiter vorn liegen“ [17, DK B1 S. 257], denn für jede Teilstrecke $t_{a}$ gibt es (potentiell) immer eine Teilstrecke mit $t_{e}<t_{a}$ beispielsweise $t_{e}=t_{(a 0)}$ und kein Maximum, keine letzte Teilstrecke, „kein solches von ihm wird ein Letztes sein“ [17, DK B1 S. 257], denn (potentiell) gibt es immer eine Teilstrecke mit $t_{a}<t_{e}$, beispielsweise $t_{e}=t_{(a 1)}$ (für einem Teilungspunkt in $t_{a}$ ).

Das mathematische Modell der beliebig lang fortgesetzten endlichen Teilung ist eine nicht leere, abzählbar unendliche, unbeschränkte und dichte linear geordnete Menge von Teilstrecken. Die Menge dieser Teilstrecken ist folglich ordnungsisomorph zu den rationalen Zahlen $(\mathbb{Q},<)$ (Satz 1).

\subsection{Abbildung auf den Zahlenstrahl}

Es bleibt die grafische Darstellung dieser Teilstrecken genauer zu betrachten. Die Wahl eines geeigneten Maßstabs für jede Teilstrecke ist entscheidend. Halten wir den Maßstab für die Gesamtstrecke fest und stellen alle Teilstrecken proportional dar, dann ergibt sich (zeitlich interpretiert) das Bild eines sich ständig verändernden Kontinuums, mit einer Dynamik des Vergehens und Entstehens von Teilen (wie in Abb. 3). 


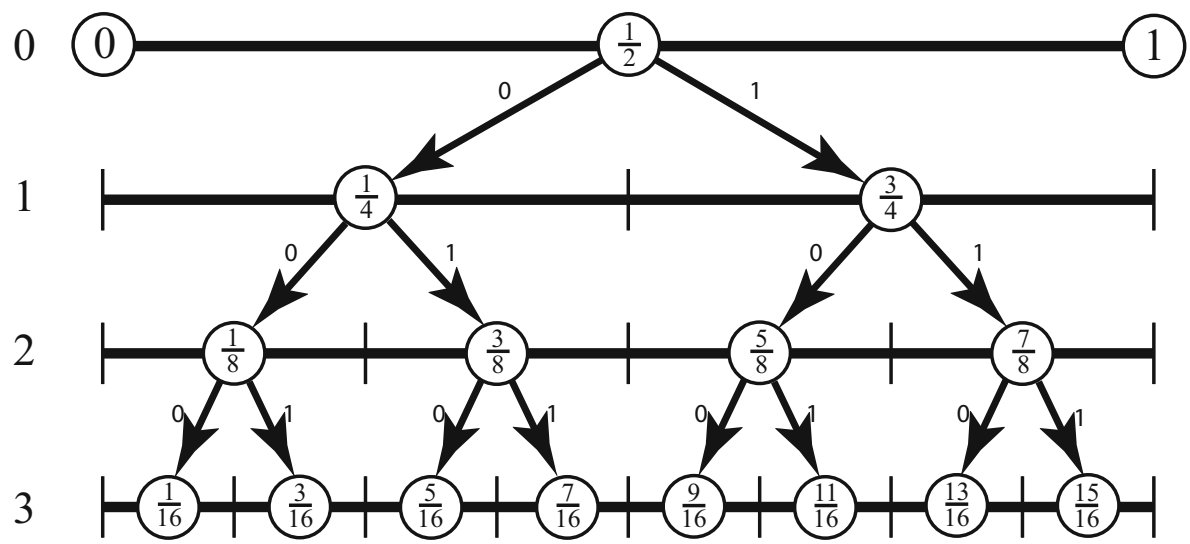

Abb. 4 Proportionale Abbildung der Punkte

Dabei bilden wir die Punkte auf den rationalen Zahlenstrahl ab. Einem Punkt $P_{a}$ mit dem Code $\left(a_{i}\right)_{i \in[0, n)}$ können wir den Zahlenwert $q_{a} \in \mathbb{Q}$ zuordnen, indem wir die Binärfolge als dyadischen Bruch $\frac{z}{2^{m}}$ interpretieren:

$$
\widetilde{q}_{a}:=1-\frac{1}{2}+\sum_{i=0}^{n-1} \frac{(-1)^{1+a_{i}}}{2^{i+2}}
$$

Dem Anfangspunkt $A$ der Gesamtstrecke $\left.t_{(}\right)=[A Z]$ weisen wir den Wert 0 , dem Endpunkt $Z$ den Wert 1 und ihrem Teilungspunkt $O$ den Wert $\frac{1}{2} \mathrm{zu}$ und erhalten so Werte $0<\widetilde{q}_{a}<1$ (die Werte für Folgen $\left(10 a_{0} \ldots a_{n-1}\right)$ in Definition 3). Der dyadische Baum der Teilstrecken (Abb. 3) lässt sich so grafisch proportional darstellen (Abb. 4).

Wir analysieren primär die Ordnungsstruktur der Teilstrecken, daher ist kein einheitlicher Maßstab oder Verhältnis vorgegeben. Aus Gründen, die im Abschn. 6.1 über surreale Zahlen deutlicher werden, wählen wir für die jeweils äußeren Teilstrecken denselben Maßstab wie für die ungeteilte Strecke und nur für innere Teilstrecken den proportionalen Maßstab wie zuvor.

Definition 3 Für eine endliche Binärfolge $a=\left(a_{i}\right)_{i \in[0, n)}$ bezeichnet der Index $k_{a}=\min (\{0 \leq i<n \mid a(0) \neq a(i)\} \cup\{n-1\})$ die Stelle des ersten Wechsels und wir können $a$ den dyadischen Bruch:

$$
q_{a}=Q(a):=(-1)^{1+a(0)} \cdot k_{a}+\sum_{k_{a} \leq i<n} \frac{(-1)^{1+a(i)}}{2^{i-k_{a}+1}}
$$

zuordnen, wobei wir der leeren Folge $\left.q_{(} \quad\right)=0$ zuweisen [12, Abschn. 4 A-C].

Bilden wir den Punkt $P_{a}$ mit Code $a$ auf den Wert $q_{a}$ ab und den Teilungspunkt $O$ der Gesamtstrecke $[A Z]$ auf den Wert 0, dann erhalten wir alle rationalen Zahlen mit endlicher dyadischer Darstellung als Bildpunkte (Abb. 5). Zeitlich interpretiert ergibt 


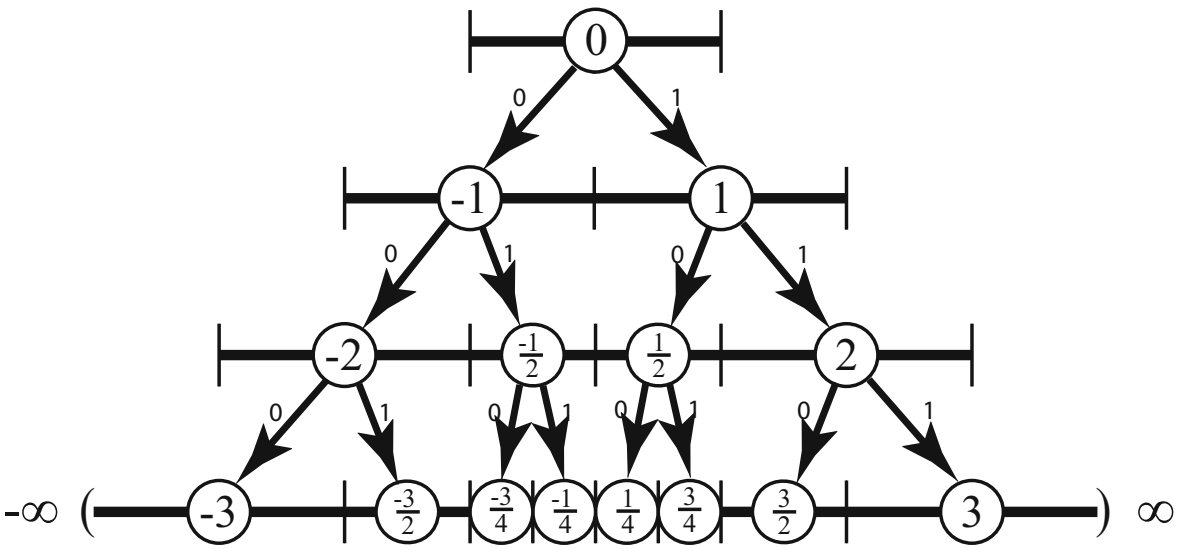

Abb. 5 Baum der endlichen surrealen Zahlen

sich das Bild eines linear nach außen und fraktal in seine Teile hinein wachsenden Baumes.

\section{Unendliche Teilungen}

Die geordnete Menge der Teilungspunkte beliebig langer endlicher Teilungen ist dicht bezüglich der Ordnung, jedoch nicht vollständig. Ist $(\overline{10})$ die unendliche Folge mit Periode 10, dann ist der Schnitt $L:=\left\{P_{a} \mid a \leq \overline{(10)}\right\}$ und $R:=\left\{P_{a} \mid a>(\overline{10})\right\}$ eine Lücke und die Intervallschachtelung aus Beispiel 1 (s.u.): $\left.I_{0}:=\left[P_{(}\right), P_{(1)}\right]$, $I_{1}:=\left[P_{(10)}, P_{(1)}\right], I_{2}:=\left[P_{(10)}, P_{(101)}\right], \ldots$ enthält keinen gemeinsamen Punkt (denn $\frac{2}{3}=(0.10)_{2}$ hat keine endliche dyadische Darstellung). Daher wollen wir ab jetzt unbeschränkte, aktual unendliche Teilungen zulassen und benötigen passende Namen und Zahlen. Im Endlichen haben wir gesehen: Binärfolgen eignen sich zur Bezeichnung der Teilstrecken und dyadische Zahlen als Bilder der Punkte. Im Unendlichen verwenden wir unendlich lange Binärfolgen und surreale Zahlen.

\subsection{Surreale Zahlen}

Surreale Zahlen und ihre Ordnung wurden von Conway rekursiv konstruiert [7, Nullter Teil], [16]. Surreale Zahlen lassen sich als verallgemeinerte dedekindsche Schnitte $(L \mid R)$ auffassen, d.h. als Paare von Mengen $L$ und $R$, die allerdings weder nur abzählbar oder gar endlich sein müssen. Die Grundideen sind recht einfach und einleuchtend, die genaue Durchführung des öfteren ziemlich mühsam - uns interessiert vor allem die Idee [13, Kap. 13].

Definition 4 Sind $L$ und $R$ zwei beliebige (möglicherweise leere) Mengen von bereits definierten surrealen Zahlen und gibt es keine Elemente $x^{L} \in L$ und $x^{R} \in R$ 
mit $x^{R} \leq x^{L}$, dann ist $z:=(L \mid R)$ eine surreale Zahl. Alle surrealen Zahlen $z=\left(L_{z} \mid R_{z}\right) \in$ No entstehen auf diese Weise.

Anmerkung 1 Werden Mengen durch Aufzählung beschrieben, dann schreiben wir die Mengenklammer in den Komponenten einer surrealen Zahl nicht unbedingt, wenn diese eindeutig lesbar bleibt, beispielsweise $\left(\left\{\frac{1}{4}\right\} \mid\{3\}\right)=\left(\frac{1}{4} \mid 3\right)$.

Mit den von-Neumann-Ordinalzahlen $(\mathrm{On},<)[18, \S 19, \S 25]$ :

$$
0:=\varnothing, 1:=\{0\}, 2:=\{0,1\}, \ldots, \omega:=\{0,1,2, \ldots\}, \ldots, \alpha:=\{\beta \mid \beta<\alpha\}, \ldots
$$

erhalten wir sofort eine ganze echte Klasse von surrealen Zahlen:

$$
0:=(\varnothing \mid \varnothing), 1:=(0 \mid \varnothing), \ldots, \omega:=(0,1,2, \ldots \mid \varnothing), \ldots, \alpha:=(\{\beta \mid \beta<\alpha\} \mid \varnothing), \ldots
$$

[7, S. 10], [13, S. 292].

Definition 5 Sind $x=\left(L_{x} \mid R_{x}\right) \in$ No und $y=\left(L_{y} \mid R_{y}\right) \in$ No zwei surreale Zahlen, dann gilt $x \leq y$ genau dann, wenn es kein Element $y^{R} \in R_{y}$ mit $y^{R} \leq x$ und keine Element $x^{L} \in L_{x}$ mit $y \leq x^{L}$ gibt. $x=y$ gilt genau dann, wenn $x \leq y$ und $y \leq x$ gilt. $x<y$ gilt genau dann, wenn $x \leq y$ gilt und $y \leq x$ nicht gilt.

Anmerkung 2 Hier wird neben der Ordnung auch die Gleichheit von surrealen Zahlen definiert und von der formalen Identität unterschieden, beispielsweise $(0 \mid \varnothing)=\left(\frac{1}{4} \mid 3\right)[7$, S. 12]

Für jede surreale Zahl $x \in$ No und für alle Zahlen $x^{L} \in L_{x}$ gilt $x^{L}<x$ und für alle Zahlen $x^{R} \in R_{x}$ gilt $x<x^{R}$.

Satz 3 Die surrealen Zahlen zusammen mit dieser Ordnung $(\mathrm{No},<)$ bilden eine strikt linear geordnete echte Klasse.

Anmerkung 3 Sei $L$ eine nach oben und $R$ eine nach unten beschränkte Menge surrealer Zahlen. Existieren $\max (L)$ und $\min (R)$ nicht, dann existieren $\sup (L)$ und $\inf (R)$ in $(\mathrm{No},<)$ ebenfalls nicht; denn wäre $s=\sup (L)$, dann wäre die Zahl $(L \mid\{s\})$ eine kleinere obere Schranke von $L$ und wäre $i=\inf (R)$, dann wäre die Zahl $(\{i\} \mid R)$ eine größere untere Schranke von $R$.

Auf $($ No, $<)$ kann man rekursiv die Struktur eines angeordneten, kommutativen Körpers definieren [7, Nullter Teil], dieser Körper ist nicht-archimedisch und enthält infinit große und infinitesimal kleine Zahlen.

\subsection{Unendliche Binärfolgen}

Mit Hilfe von Ordinalzahlen lassen sich surreale Zahlen in äquivalenter Weise als unendliche Folgen auffassen [12]. Für $\alpha \in$ On ist $[0, \alpha)=\{\beta \in$ On $\mid \beta<\alpha\}$. 
Definition 6 Die (echte) Klasse der binären Folgen bezeichnen wir mit

$$
\mathrm{Seq}_{2}:=\{a:[0, \alpha) \rightarrow\{0,1\} \mid \alpha \in \mathrm{On}\}
$$

und mit $l(a):=\alpha$ die Länge solcher Folgen.

Die Folge $b$ heißt Anfangsstück der Folge $a$ im Zeichen $b=a \mid[0, \beta)$, wenn $l(b)=\beta<l(a)$ und $b(\gamma)=a(\gamma)$ für alle $\gamma<\beta$ gilt und dies definiert eine Baumordnung $b<_{S} a$ auf $\operatorname{Seq}_{2}$. Sind $a, b \in \operatorname{Seq}_{2}$ mit $l(a)=\alpha$ und $l(b)=\beta$, dann betrachten wir die Stelle des ersten Unterschieds

$$
\delta_{a, b}:=\min (\{\gamma \in \mathrm{On} \mid \gamma<\alpha \text { und } \gamma<\beta \text { und } a(\gamma) \neq b(\gamma)\} \cup\{\alpha, \beta\})
$$

und definieren eine totale Ordnung der Folgen durch $a<b$ genau dann, wenn $a\left(\delta_{a, b}\right)=0$ (falls definiert) und $b\left(\delta_{a, b}\right)=1$ (falls definiert). Die Folge $c_{a, b}:=$ $a\left|\left[0, \delta_{a, b}\right)=b\right|\left[0, \delta_{a, b}\right)$ ist das gemeinsame Anfangsstück von $a$ und $b$. Die Struktur $\left(\mathrm{Seq}_{2},<_{S},<\right)$ ist ein geordneter dyadischer Wurzelbaum mit der leeren Folge als Wurzel $r=(\quad)$.

Satz $4 Z u$ zwei Mengen $L, R \subset \mathrm{Seq}_{2}$ binärer Folgen mit $L<R$ gibt es eine kürzeste Folge c mit $L<c<R$; wobei kürzeste Folge heißt, dass für alle $L<s<R$ gilt $c=s \mid[0, l(c)), d$.h. $c$ ist das gemeinsame Anfangsstück aller solcher Folgen [12, Theorem 2.1]. Wir setzen $(L \mid R):=c$.

Entscheidend ist hier die Wahl des einfachsten Elements. Daraus folgt, dass falls $a=\max (L)$ und $b=\min (R)$ existieren, gilt $(L \mid R)=(a \mid b)=c$. Etwas schwerer folgt die Existenz einfacher kanonischer Repräsentanten, die wir als Schachtelung von Intervallen interpretieren werden [12, Theoreme 2.6, 2.7].

Satz 5 (Kanonische Repräsentanten) Umgekehrt können wir jeder Folge $c \in$ $\mathrm{Seq}_{2}$ auf natürliche Weise zwei Mengen $L_{c}<R_{c}$ zuordnen:

$$
\begin{array}{ll}
L_{c}:=\{a \mid a<c \text { und } a=c \mid[0, \alpha) & \text { für ein } \alpha<l(c)\} \subset \mathrm{Seq}_{2} \\
R_{c}:=\{b \mid b>c \text { und } b=c \mid[0, \beta) & \text { für ein } \beta<l(c)\} \subset \mathrm{Seq}_{2}
\end{array}
$$

dann gilt $\left(L_{c} \mid R_{c}\right)=c$. [12, Theorem 2.8]

Nun lassen sich zwei Funktionen rekursiv definieren:

$$
\begin{aligned}
& F: \mathrm{No} \longrightarrow \mathrm{Seq}_{2} \\
& z=\left(L_{z} \mid R_{z}\right) \mapsto c:=\left(F\left(L_{z}\right) \mid F\left(R_{z}\right)\right)
\end{aligned}
$$

mit $F((\varnothing \mid \varnothing)):=(\quad)$ und $c$ die kürzeste binäre Folge mit $F\left(L_{z}\right)<c<F\left(R_{z}\right)$ wie in Satz 4 [12, S. 7]. Umgekehrt definieren wir

$$
\begin{aligned}
& G: \mathrm{Seq}_{2} \longrightarrow \mathrm{No} \\
& c \mapsto\left(G\left(L_{c}\right) \mid G\left(R_{c}\right)\right)
\end{aligned}
$$

rekursiv über die Länge von Folgen, wobei $G((\quad)):=(\varnothing \mid \varnothing)$ ist. 
Diese Funktionen $F$ und $G$ sind Inverse, d.h. $G(F(z))=z$ für alle $z \in$ No und $F(G(c))=c$ für alle $c \in \mathrm{Seq}_{2}$ [12, Theorem 2.8] und ordnungstreu [12, Theorem 2.5] - also Isomorphismen geordneter Klassen. Mittels der Funktion $F$ lässt sich auch die Ordnung $\left(\mathrm{Seq}_{2},<_{S}\right)$ auf No zurückziehen; d.h. $y<_{S} x$ gilt genau dann, wenn $F(y)<{ }_{S} F(x)$ gilt. Damit ist auch $\left(\right.$ No, $\left.<_{S},<\right)$ ein geordneter dyadischer Wurzelbaum mit der Wurzel $r=(\varnothing \mid \varnothing)$. Der Geburtstag einer surrealen Zahl $z \in$ No ist dann die Länge der zugehörigen Folge $\operatorname{rg}_{<_{S}}(z):=l(F(z)) \in$ On $[18, \S$ 28], [12, Theorem 2.3], [7, Satz 16].

\subsection{Unendliche arithmetische Teilungen}

Zur Definition aktual unendlicher Teilungen braucht man einen passendes Verständnis von Grenzstrecken und deren Grenzpunkten. Wir wählen einen Limes-Begriff, der den aristotelischen Begriff erweitert [19, S. 23ff.]. Den ordnungstheoretischen Betrachtungen angemessen sind die Begriffe Maximum, Supremum, Minimum, Infimum und Intervallschachtelung.

Zuerst wollen wir die Teilungen an möglichst intuitiven Beispielen von Intervallschachtelungen im geordneten $\operatorname{Körper}(\mathbb{Q},<)$ untersuchen. Wir betrachten das rationale Intervall $\left.I_{(}\right)=[0,1]=\{x \in \mathbb{Q} \mid 0 \leq x \leq 1\}$. Im Beispiel 1 erhalten wir einen Punkt als Grenzintervall, im Beispiel 2 die leere Menge und in Beispiel 3 ein Intervall endlicher, positiver Länge. In allen Beispielen wenden wir das gleiche Teilungsschema nur mit unterschiedlichen Punkten an.

Beispiel 1 Wir teilen immer bei dyadischen Brüchen $\frac{z}{2^{m}}$. Zuerst teilen wir das Einheitsintervall bei der Zahl $q_{1}=\frac{1}{2}$ in die zwei Teilintervalle $I_{(0)}=\left[0, \frac{1}{2}\right]$ und $I_{(1)}=\left[\frac{1}{2}, 1\right]$. Anschließend teilen wir das hintere Teilintervall $I_{(1)}$ bei der Zahl $q_{2}=\frac{1}{2}+\frac{1}{4}=\frac{3}{4}$ und nehmen das entstehende vordere Teilintervall, das wir bei der Zahl $q_{3}=\frac{1}{2}+\frac{1}{4}-\frac{1}{8}$ teilen und danach das entstehende hintere Teilintervall weiter teilen. Teilen wir immer weiter bei $q_{n}:=\sum_{i=0}^{n} \frac{(-1)^{i}}{2^{i}}$, dann erhalten wir eine absteigende Folge von Teilintervallen:

$$
\left.I_{(} \quad\right)=[0,1] \supset I_{(1)}=\left[\frac{1}{2}, 1\right] \supset I_{(10)}=\left[\frac{1}{2}, \frac{3}{4}\right] \supset I_{(101)}=\left[\frac{5}{8}, \frac{3}{4}\right] \supset \ldots
$$

und das Grenzintervall $I_{\infty}=\lim _{n \rightarrow \infty} I_{n}=\bigcap_{n=1}^{\infty} I_{n}=\left[\frac{2}{3}, \frac{2}{3}\right]=\left\{\frac{2}{3}\right\}$.

Beispiel 2 Nehmen wir die Fibonacci-Folge $\left(f_{n}\right)=(3,5,8,13,21, \ldots)$ und teilen bei den Zahlen $q_{n}:=\frac{f_{n}}{f_{n+1}}$, dann erhalten wir:

$$
\left.I_{(} \quad\right)=[0,1] \supset I_{(1)}=\left[\frac{3}{5}, 1\right] \supset I_{(10)}=\left[\frac{3}{5}, \frac{5}{8}\right] \supset I_{(101)}=\left[\frac{8}{13}, \frac{5}{8}\right] \supset \ldots
$$

mit Grenzintervall $I_{\infty}=\varnothing$ (die Intervallschachtelung approximiert das Reziproke $\frac{1}{\Phi}$ der Goldenen Zahl $\left.\Phi\right)$. 
Beispiel 3 Teilungen bei $q_{1}=\frac{1}{4}, q_{2}=\frac{3}{4}, q_{3}=\frac{5}{16}, q_{5}=\frac{11}{16}, \ldots$ ergeben:

$$
\left.I_{(} \quad\right)=[0,1] \supset I_{(1)}=\left[\frac{1}{4}, 1\right] \supset I_{(10)}=\left[\frac{1}{4}, \frac{3}{4}\right] \supset I_{(101)}=\left[\frac{5}{16}, \frac{3}{4}\right] \supset \ldots
$$

mit Grenzintervall $I_{\infty}=\left[\frac{1}{3}, \frac{2}{3}\right] \subseteq \mathbb{Q}$.

\subsection{Unendliche geometrische Teilungen}

Das Teilbarkeitsprinzip (Definition 2) gelte nun auch bei einer unbeschränkt ins unendliche fortgesetzten Teilung einer kontinuierlichen, teilbaren Strecke; das Beispiel 3 ist exemplarisch auch für aktual unendliche Teilungen.

\section{Definition 7 (Prinzip der unbeschränkten Teilung)}

Ist jede Strecke einer unendlichen Streckenfolge eine teilbare, kontinuierliche Strecke und Teil der vorhergehenden Strecken, so gibt es eine teilbare, kontinuierliche Strecke, die Teil aller Strecken der Folge ist.

Zuerst fordern wir in einer rekursiven Definition die Existenz von Grenzpunkten zwischen bereits definierten Punkten vergleichbar der Definition von surrealen Zahlen. Wir wählen eine Orientierung der Strecke $[A Z]$ durch $A<Z$.

\section{Definition 8 (Punkte der unbeschränkten Teilung)}

Sind $L$ und $R$ zwei Mengen von bereits definierten Punkten auf $[A Z]$ und gibt es kein $P^{L} \in L$ und kein $P^{R} \in R$ mit $P^{R} \leq P^{L}$, dann gibt es einen Punkt $P:=(L \mid R)$ auf $[A Z]$. Für zwei solche Punkte $P=\left(L_{P} \mid R_{P}\right)$ und $Q=\left(L_{Q} \mid R_{Q}\right)$ gilt $Q \leq P$ genau dann, wenn es kein $P^{R} \in R_{P}$ mit $P^{R} \leq Q$ und kein $Q^{L} \in L_{Q}$ mit $P \leq P^{L}$ gibt.

Anmerkung 4 Diese Punkte Pt und die surrealen Zahlen werden strukturgleich definiert, daher lässt sich auf natürliche Weise ein Ordnungsisomorphismus von den Punkten $(\mathrm{Pt},<)$ auf die surrealen Zahlen $(\mathrm{No},<)$ rekursiv definieren:

$$
\begin{aligned}
& H: \mathrm{Pt} \longrightarrow \text { No } \\
& P \mapsto\left(H\left(L_{P}\right) \mid H\left(R_{P}\right)\right) .
\end{aligned}
$$

Für $(\mathrm{Pt},<)$ gilt daher wie für $(\mathrm{No},<)$ : Sind $L$ eine nach oben und $R$ eine nach unten beschränkte Menge von Punkten, für die $\max (L)$ und $\min (R)$ nicht existieren, dann existieren $\sup (L)$ und $\inf (R)$ ebenfalls nicht.

Dem Charakter einer fortgesetzten Zweiteilung entsprechend definieren wir Teilstrecken von $[A Z]$ mit Hilfe einer Nachfolger-Limes-Rekursion für Ordinalzahlen [18, S. 183] und bezeichnen diese mit $t_{a}$ für eine Binärfolge $a \in \mathrm{Seq}_{2}$ der Länge $l(a) \in$ On. Gleichzeitig definieren wir eine partielle Ordnung $<_{s}$, wobei $s<_{s} t$ bedeuten soll: „t ist Teilstrecke von $s$ “. Ketten dieser Ordnung sind ineinandergeschachtelte Teilstrecken und ihr Limes wird als Grenzwert dieser Streckenschachtelung definiert. 


\section{Definition 9 (Strecken der unbeschränkten Teilung)}

1. Sei $\alpha=0 \in$ On, dann ist $t_{(} \quad$ ) $:=[A Z]$ die Gesamtstrecke, die als kontinuierlich und teilbar vorausgesetzt wurde.

2. Sei $\alpha=\beta+1 \in$ On eine Nachfolgerzahl und $t_{b}=[Q S]$ mit $l(b)=\beta$ eine bereits definierte, teilbare, kontinuierliche Teilstrecke von $[A Z]$ und wählen wir einen Teilungspunkt $P_{b}:=P=(Q \mid S)$ mit $Q<P<S$, dann sind $t_{(b 0)}:=[Q P]$ und $t_{(b 1)}:=[P S]$ teilbare, kontinuierliche Teilstrecken von $[A Z]$ und $t_{b}$ mit Länge $\alpha$. Wir setzen $t_{b}<s t_{(b 0)}, t_{(b 1)}$.

3. Sei $\alpha=\lambda \in$ On eine Limeszahl und $\left(t_{a_{\alpha}}\right)_{\alpha<\lambda}$ eine $<_{S}$-Kette von bereits definierten, kontinuierlichen, teilbaren Teilstrecken $t_{a_{\alpha}}=\left[Q_{\alpha} S_{\alpha}\right]$ von $[A Z]$ mit $t_{a_{\alpha}}<{ }_{S} t_{a_{\beta}}$ für alle $\alpha<\beta<\lambda$. Für die Mengen $L:=\left\{Q_{\alpha} \mid \alpha<\lambda\right\}$ und $R:=$ $\left\{S_{\alpha} \mid \alpha<\lambda\right\}$ wählen wir zwei Punkte $Q$ und $S$ mit $L \leq Q<S \leq R$. Sei $a \in \operatorname{Seq}_{2}$ die Folge mit $a_{\alpha}:=a \mid[0, \alpha)$ für alle $\alpha<\lambda$, dann ist $t_{a}:=[Q S]$ eine teilbare, kontinuierliche Teilstrecke von $[A Z]$ und allen $t_{a_{\alpha}}$. Wir setzen $t_{a}<s_{S} t_{(}, t_{a_{\alpha}}$ für alle $\alpha<\lambda$. Falls existent wählen wir $Q=\max (L)$ und $S=\min (R)$, ansonsten $Q=(L \mid R)$ und $S=(Q \mid R)$.

Eine totale Ordnung der Teilstrecken definieren wir durch $t_{a}<t_{b}$ genau dann, wenn $a<b$ gilt.

Die Klasse Ts der durch eine unbeschränkt fortgesetzte Zweiteilung konstruierten Teilstrecken von $[A Z]$ zusammen mit der partielle Ordnung $<_{S}$ und der totalen Ordnung $<$ definiert die Struktur $\left(\mathrm{Ts},<_{S},<\right)$ eines geordneten dyadischen Wurzelbaums. Die Funktion $T: \mathrm{Ts} \rightarrow \mathrm{Seq}_{2}$ definiert durch $t_{a} \mapsto a$ ist ein Isomorphismus von geordneten dyadischen Wurzelbäumen. Die Relation $<_{S}$ ist fundiert und ihre Rangfunktion $\operatorname{rg}_{<_{S}}(t):=l(T(t))$ definiert eine Ranghierarchie auf Ts $[18, \S 28]$.

\section{Paradoxien der Teilung}

\subsection{Anzahl der Teile und Selbstähnlichkeit}

Wie viele Teilstrecken gibt es? Zenon schreibt: „Wenn (die Dinge) viele sind, ist es zwingend, dass sie eben so viele sind, wie sie sind, und weder mehr als sie noch weniger." [17, DK B3 S. 257]. Aber weder ist die Klasse der durch eine unbeschränkt fortgesetzte Zweiteilung konstruierten Teilstrecken noch die Klasse der Teilungspunkte gleichzahlig zu einer Ordinalzahl, ihre Anzahl ist die Anzahl einer geordneten echten Klasse.

Die ganze Strecke ist ihren Teilen ähnlich, denn jede Folge $c \in \mathrm{Seq}_{2}$ lässt sich durch beliebige Folgen $d \in \mathrm{Seq}_{2}$ zu einer Folge $c<_{S}(c d) \in \mathrm{Seq}_{2}$ verlängern. Die Abbildung $c \mapsto(c d)$ ist injektiv. Ist $t_{c}=[Q S]$ eine Teilstrecke von $[A Z]$, dann sind alle durch fortgesetzte Teilung entstehenden Teilstrecken von $[Q S]$ von der Form $t_{(c d)}$. Die Teilstrecken von $[A Z]$ lassen sich also durch einen OrdnungsIsomorphismus auf die Teilstrecken von $[Q S]$ abbilden und beide haben die Anzahl einer echten Klasse. 


\subsection{Infinitesimale Strecken}

Infinitesimale sind ein metrischer bzw. algebraischer Begriff. Wir verwenden hier die Körperstruktur der surrealen Zahlen. Die Folgen (10...0) der Länge $1+m$ können mittels der Abbildung $Q$ (Definition 3) mit den dyadischen Brüchen $\frac{1}{2^{m}}$ identifiziert werden [7, S. 19], [12, Theorem 4.2].

Der angeordnete Körper der Zahlen $\left(\mathrm{Seq}_{2},<\right)$ enthält dann positive infinitesimale Zahlen. Sei $\epsilon \in \mathrm{Seq}_{2}$ mit $l(\epsilon)=\omega$ die Binärfolge $\epsilon(0)=1$ und $\epsilon(i)=0$ für alle $0 \neq i<\omega$. Es gilt $0<\epsilon<\frac{1}{2^{m}}$ für alle $m$; d.h. $\epsilon$ ist eine infinitesimal kleine Zahl relativ zu diesen dyadischen Brüchen [12, S. 48], [11, S. 506f.].

$I:=\left\{\iota \in \mathrm{Seq}_{2} \mid 0<\iota<\frac{1}{2^{m}}\right.$ für alle $\left.m \in \mathbb{N}\right\}$ sei die Klasse aller positiven Infinitesimalen. Für jedes $d \in \mathrm{Seq}_{2}$ gilt $0<(\epsilon d)<\frac{1}{2^{m}}$ für alle $m \in \mathbb{N}$ und damit $(\epsilon d) \in I$. Somit ist auch das Intervall $I$ eine echte Klasse. Die linke Grenze des Intervalls $I=(a, b)$ ist $a=0$ und die rechte Grenze ist der Schnitt $\frac{1}{\infty}:=$ $\left(I \mid\left\{\frac{1}{2^{m}} \mid m \in \mathbb{N}\right\}\right)$; dieser Schnitt ist jedoch keine Zahl, denn $I$ ist keine Menge. Der Schnitt $\frac{1}{\infty}$ ist eine Lücke von No

Bei der Abbildung $H$ der Punkte auf den Zahlenstrahl der surrealen Zahlen werden alle Punkte $P_{\iota}$ mit $\iota \in I$ auf positive infinitesimale Zahlen abgebildet und alle Teilstrecken $t_{\iota}$ auf infinitesimale Strecken. Die Grenze zwischen den Punkten mit positiv infinitesimalen Werten und den Punkten mit positiv finiten Werten ist eine Lücke.

\subsection{Limites und Grenzen}

Betrachten wir die arithmetischen Teilungen des Einheitsintervalls wie in den Beispielen 1, 2 und 3 diesmal in den surrealen Zahlen $[0,1] \subset$ No, so erhalten wir als Limites $\lim _{n \rightarrow \infty} I_{n}=\bigcap_{n=1}^{\infty} I_{n}$ für diese Intervallschachtelungen:

$$
\left[\frac{2}{3}-\frac{1}{\infty}, \frac{2}{3}+\frac{1}{\infty}\right],\left[\frac{1}{\Phi}-\frac{1}{\infty}, \frac{1}{\Phi}+\frac{1}{\infty}\right],\left[\frac{1}{3}-\frac{1}{\infty}, \frac{2}{3}+\frac{1}{\infty}\right] \text {. }
$$

Bei einer Abbildung auf den reellen Zahlenstrahl, die die Abbildung $Q$ aus Definition 3 stetig fortsetzt, würden alle drei Grenzintervalle auf $\left[\frac{1}{2}, \frac{1}{2}\right]$ abgebildet, d.h. sie sind alle infinitesimal. Dass die Punkte zuvor bereits als Zahlen interpretiert waren, wird in der Ordnungstheorie natürlich vergessen.

Die Grenzen dieser Intervalle sind keine surrealen Zahlen, sie sind Lücken der surrealen Zahlen. Tatsächlich gibt es in den surrealen Zahlen $(\mathrm{No},<)$ sehr viele Lücken, ihre Ansammlung ist nicht einmal eine echte Klasse [7, S. 32]. Die Lücken sind allerdings interessant und einige haben eine analysierbare Struktur [7, Kap. 16].

Eine Lesart des viel diskutierten zenonschen Satzes „Aus diesem Grund ist es, wenn (die Dinge) viele sind, zwingend, dass sie sowohl klein als auch groß sind; so klein, dass sie keine Ausdehnung haben dürften, und so groß, dass sie unendlich sein müssten." [17, DK B1 S. 257] wäre somit die, dass es zu jeder Teilstrecke $t$ einer teilbaren, kontinuierlichen Strecke eine Teilstrecke $t^{\prime}$ gibt, die infinitesimal klein relativ zu $t$ ist (d.h. $n t^{\prime}<t$ für alle $n \in \mathbb{N}$ ) und es eine Teilstrecke $s$ gibt, die infinit groß relativ zu $t$ ist (d.h. $n t<s$ für alle $n \in \mathbb{N}$ ). Die Begriffe ,groß“ und „klein“ 
sind ja relationale Begriffe, die erst durch die Wahl einer Struktur festgelegt werden; dies rechtfertigt auch die Veranschaulichung durch „Unendlichkeitslupen“ [5, S. 424]. In den surrealen Zahlen gibt es zu jedem archimedischen Zahlenbereich viele infinitesimal kleinere und infinit größere Zahlenbereiche, deren Grenzen Lücken sind [7, Kap. 16].

\section{Fazit}

Im klassenlogischen Rahmen der allgemeinen Mengenlehre ist es uns gelungen drei ordnungstheoretische Modelle für die Teilstrecken einer geometrische Strecke zu konstruieren, die zu den drei klassischen Auffassungen des Kontinuums von Parmenides, Aristoteles und Zenon passen.

Die Vorstellung des Parmenides vom unteilbaren „Seienden“ entspricht dem sehr einfachen Modell einer Einermenge - dieses Modell ist provokant einfach. Um Modelle mit mehr Struktur zu erhalten, haben wir die Strecke an Punkten geteilt und sind dabei dem einfachen Teilbarkeitsprinzip der Definition 2 gefolgt, was unmittelbar zu unendlich vielen Teilstrecken führt.

Aristoteles potentiell unendlich, aktual nur endlich oft teilbares Kontinuum, entspricht einem Modell, bei dem die Ordnung der Teile Lücken hat. Die benachbarten Teilstrecken einer aktualisierten endlichen Teilung hängen allerdings immer lückenlos in einem Punkt zusammen. Die potentiell unendlich vielen Punkte sind abzählbar und werden durch Paare $(L \mid R)$ endlicher Mengen bereits konstruierter Punkte bestimmt.

Zenons hypothetisches Kontinuum, das aktual unendlich oft teilbare Kontinuum, entspricht einem Modell, das dem unbeschränkten Teilbarkeitsprinzip der Definition 7 genügt. Die Klasse der Punkte ist dabei isomorph zu den surrealen Zahlen, dem universellen, absoluten, arithmetischen Kontinuum [11, Surreal 6, S. 530]. Diese Punkte werden durch Paare $(L \mid R)$ beliebig großer Mengen bereits konstruierter Punkte bestimmt und bilden eine echte Klasse.

Auch diese Ordnung hat Lücken; allerdings ist sie intervallvollständig, für nichtarchimedische Geometrien wohl ein angemessenerer Begriff [11, S. 509]. Schon Hilbert weißt darauf hin, dass sein Vollständigkeitsaxiom ohne das archimedische Axiom einen Widerspruch einschließen würde [14, §8 S. 31]. Leider geht der Zusammenhang von Teilstrecken in Limes-Schritten verloren.

Die Lücken gehören zu Paaren $(L \mid R)$ echter Klassen. Wenn diese Paare ebenfalls Punkte definieren sollen um die Lücken zu schließen, dann gibt es noch sehr viel mehr Punkte und wir benötigen einen neuen formalen Rahmen.

Die Frage „Was ist das Kontinuum?“ bleibt also weiterhin philosophisch und mathematisch interessant und wird wohl nie abschließend befriedigend beantwortet werden können [11, S. 553f.].

Funding Open Access funding enabled and organized by Projekt DEAL.

Open Access Dieser Artikel wird unter der Creative Commons Namensnennung 4.0 International Lizenz veröffentlicht, welche die Nutzung, Vervielfältigung, Bearbeitung, Verbreitung und Wiedergabe in jeglichem Medium und Format erlaubt, sofern Sie den/die ursprünglichen Autor(en) und die Quelle ord- 
nungsgemäß nennen, einen Link zur Creative Commons Lizenz beifügen und angeben, ob Änderungen vorgenommen wurden.

Die in diesem Artikel enthaltenen Bilder und sonstiges Drittmaterial unterliegen ebenfalls der genannten Creative Commons Lizenz, sofern sich aus der Abbildungslegende nichts anderes ergibt. Sofern das betreffende Material nicht unter der genannten Creative Commons Lizenz steht und die betreffende Handlung nicht nach gesetzlichen Vorschriften erlaubt ist, ist für die oben aufgeführten Weiterverwendungen des Materials die Einwilligung des jeweiligen Rechteinhabers einzuholen.

Weitere Details zur Lizenz entnehmen Sie bitte der Lizenzinformation auf http://creativecommons.org/ licenses/by/4.0/deed.de.

Conflict of interest The author declares that he has no conflict of interest.

\section{Literatur}

1. Aristoteles: Physik Vorlesung über Natur. Zekl, H. G. (Hrsg.). Philosophische Bibliothek, Bd. 381. Meiner, Hamburg (1988)

2. Bedürftig, T.: Was ist ein Punkt? - Ein Streifzug durch die Geschichte. Siegener Beiträge zur Geschichte und Philosophie der Mathematik, Bd. 5., S. 1-21 (2015)

3. Bedürftig, T., Murawski, R.: Historische und philosophische Notizen über das Kontinuum. Math. Semesterber. 64(1), 63-88 (2017)

4. Bedürftig, T.: Über die Grundproblematik der Grenzwerte. Math. Semesterber. 65(2), 277-298 (2018)

5. Bedürftig, T., Murawski, R.: Philosophie der Mathematik, 4. Aufl. de Gruyter, Berlin München Boston (2019)

6. Bernays, P.: Betrachtungen über das Vollständigkeitsaxiom und verwandte Axiome. Math. Z. 63, 219-229 (1955)

7. Conway, H.: Über Zahlen und Spiele. Vieweg, Braunschweig (1983)

8. Deiser, O.: Reelle Zahlen, 2. Aufl. Springer, Berlin Heidelberg New York (2007)

9. Diestel, R.: Graph Theory, 5. Aufl. Springer, Berlin Heidelberg New York (2017)

10. Ehrlich, P.: All numbers great and small. In: Ehrlich, P. (Hrsg.) Real Numbers, Generalizations of the Reals, and Theories of Continua Synthese, Bd. 242, Kluwer Academic, Dordrecht (1994)

11. Ehrlich, P.: Contemporary infinitesimalist theories of continua and their late nineteenth- and early twentieth-century forerunners. In: Shapiro, S., Hellman, G. (Hrsg.) The History of Continua, Philosophical and Mathematical Perspectives. Oxford University Press, Oxford (2021)

12. Goshor, H.: An Introduction to the Theory of Surreal Numbers. Cambridge University Press, Cambridge (1986)

13. Hermes, H.: Zahlen und Spiele. In: Ebbinghaus, H.-D. (Hrsg.) Zahlen, 3. Aufl. Grundwissen Math., Bd. 1, Springer, Berlin Heidelberg New York (1992)

14. Hilbert, D.: Grundlagen der Geometrie. In: Toepell, M. (Hrsg.) Teubner-Archiv zur Mathematik, 14. Aufl. Teubner, Stuttgart Leipzig (1999)

15. Kleinert, E.: Mathematische Modelle des Kontinuums. Springer Spektrum, Berlin (2019)

16. Knuth, D.E.: Insel der Zahlen. Vieweg, Braunschweig Wiesbaden (1979)

17. Köhler, G.: Zenon von Elea. de Gruyter, Berlin München Boston (2014)

18. Oberschelp, A.: Allgemeine Mengenlehre. BI, Mannheim (1994)

19. Sattler, B.M.: Divisibility or indivisibility: the notion of continuity from the Presocratics to Aristotle. In: Shapiro, S., Hellman, G. (Hrsg.) The History of Continua, Philosophical and Mathematical Perspectives. Oxford University Press, Oxford (2021)

20. Vetter, H.: Parmenides, Sein und Welt. Karl Alber, Freiburg München (2016)

21. Wieland, W.: Das Kontinuum in der Aristotelischen Physik. In: Seeck, G.A. (Hrsg.) Die Naturphilosophie des Aristoteles. Wissenschaftliche Buchgesellschaft, Darmstadt (1975) 